\title{
LKB1 gene inactivation does not sensitize non-small cell lung cancer cells to mTOR inhibitors in vitro
}

\author{
Ping XIAO ${ }^{1}$, Lin-lin $\mathrm{SUN}^{2}$, Jing WANG ${ }^{2}$, Rui-li $\mathrm{HAN}^{1}$, Qing $\mathrm{MA}^{1}$, Dian-sheng $Z \mathrm{HONG}^{1,2, *}$ \\ ${ }^{1}$ Department of Oncology, Tianjin Medical University General Hospital, Tianjin 300052, China; ${ }^{2}$ Tianjin Lung Cancer Institute, Tianjin \\ Medical University General Hospital, Tianjin 300052, China
}

\begin{abstract}
Aim: Previous study has shown that endometrial cancers with $L K B 1$ inactivation are highly responsive to mTOR inhibitors. In this study we examined the effect of $L K B 1$ gene status on mTOR inhibitor responses in non-small cell lung cancer (NSCLC) cells.

Methods: Lung cancer cell lines Calu-1, H460, H1299, H1792, and A549 were treated with the mTOR inhibitors rapamycin or everolimus (RAD001). The mTOR activity was evaluated by measuring the phosphorylation of 4EBP1 and S6K, the two primary mTOR substrates. Cells proliferation was measured by MTS or sulforhodamine B assays.

Results: The basal level of mTOR activity in LKB1 mutant A549 and $\mathrm{H} 460$ cells was significantly higher than that in $L K B 1$ wild-type Calu-1 and H1792 cells. However, the LKB1 mutant A549 and H460 cells were not more sensitive to the mTOR inhibitors than the LKB1 wild-type Calu-1 and H1792 cells. Moreover, knockdown of LKB1 gene in $\mathrm{H} 1299$ cells did not increase the sensitivity to the mTOR inhibitors. Treatment with rapamycin or RAD001 significantly increased the phosphorylation of AKT in both LKB1 wild-type and LKB1 mutant NSCLC cells, which was attenuated by the PI3K inhibitor LY294002. Furthermore, RAD001 combined with LY294002 markedly enhanced the growth inhibition on LKB1 wild-type H1792 cells and LKB1 mutant A549 cells.

Conclusion: LKB1 gene inactivation in NSCLC cells does not increase the sensitivity to the mTOR inhibitors. The negative feedback activation of AKT by mTOR inhibition may contribute to the resistance of NSCLC cells to mTOR inhibitors.
\end{abstract}

Keywords: non small-cell lung cancer; mTOR protein; liver kinase B1; AKT; rapamycin; RAD001; LY294002

Acta Pharmacologica Sinica (2015) 36: 1107-1112; doi: 10.1038/aps.2015.19; published online 1 June 2015

\begin{abstract}
Introduction
Lung cancer is the leading cause of cancer-related deaths worldwide ${ }^{[1]}$. Mutations in tumor suppressor genes are known to play key roles in the progression of lung cancer. $L K B 1$, the causative gene for Peutz-Jeghers syndrome characterized by gastrointestinal polyposis, is frequently mutated in lung adenocarcinomas ${ }^{[2]}$. LKB1 possesses a variety of tumor-suppressive functions, such as cell polarity regulation, cell cycle arrest, induction of apoptosis and inhibition of cell growth $^{[3,4]}$. LKB1 encodes a serine/threonine protein kinase that directly activates the protein that senses intracellular ATP levels-AMP-activated protein kinase (AMPK). AMPK then phosphorylates tuberin protein (TSC2) to turn on its GTPactivating protein activity to inhibit the protein Ras homolog enriched in brain (Rheb), which inhibits mammalian target of rapamycin (mTOR) signaling ${ }^{[5]}$. Thus, LKB1/AMPK negatively regulates mTOR activity.
\end{abstract}

\footnotetext{
* To whom correspondence should be addressed.

E-mail zhongdsh@hotmail.com

Received 2014-11-05 Accepted 2015-03-06
}

mTOR is at the center of cell growth and proliferation. It acts as a scaffold to recruit the downstream substrates eukaryotic initiating factor 4E Binding Protein 1 (4EBP1) and ribosomal S6 kinase (p70S6K1) ${ }^{[6]}$. Rapamycin is the first specific mTOR inhibitor to be characterized. However, its poor aqueous solubility and chemical stability restricts its application in clinical cancer therapy. Hence, a variety of rapamycin analogs (termed rapalogs) have been developed, including RAD001 (everolimus) and CCI-779 (temsirolimus) among others ${ }^{[7]}$. Previous studies have shown that rapalogs are effective in certain types of tumors ${ }^{[8,9]}$, and approximately $26 \%$ of cancers are sensitive to $\mathrm{mTOR}$ inhibition ${ }^{[10]}$. It is conceivable that various genetic backgrounds might contribute different responses to mTOR inhibition.

Because LKB1 negatively regulates mTOR activity, it is speculated that LKB1 inactivation may lead to the hyperactivation of mTOR and result in increased sensitivity to mTOR inhibitors. Accordingly, LKB1-inactivated endometrial cancers have been shown to be highly responsive to mTOR inhibition ${ }^{[11]}$. Therefore, LKB1 deficiency in tumors appears to be a predictive for good clinical responses to mTOR inhibitors. How- 
ever, in the present study, we found that LKB1 inactivation in NSCLC cells did not increase sensitivity to mTOR inhibitors. In addition, we show that mTOR inhibition induces negative feedback activation of AKT in a PI3K-dependent manner, which possibly contributes to the mTOR inhibitor resistance.

\section{Materials and methods Materials}

Antibodies against phospho-S6K-Thr389, phospho-4EBPSer65, phospho-AKT, AKT, 4EBP1, and S6K were purchased from Cell Signaling Technology (Beverly, MA, USA). Mouse anti-GAPDH antibody was purchased from Santa Cruz Biotechnology (Santa Cruz, CA, USA). Rapamycin, RAD001 (everolimus, an allosteric mTOR inhibitor) and LY294002 were purchased from LC Laboratories (Boston, MA, USA). Sulforhodamine B (SRB) was purchased from Sigma-Aldrich (St Louis, MO, USA).

\section{Cell lines and cell culture}

Five lung cancer cell lines: Calu-1, H460, H1299, H1792, and A549 were purchased from the American Type Culture Collection (ATCC, Manassas, VA, USA). LKB1 stable knock down cell line H1299-LKB1shRNA and control cell line H1299PLKO.1 were established in our lab ${ }^{[12]}$. Cells were cultured in RPMI-1640 medium (Gibco, Grand Island, NY, USA) supplemented with $10 \%$ fetal bovine serum (Gibco) at $37^{\circ} \mathrm{C}$ in a humidified atmosphere with $5 \% \mathrm{CO}_{2}$.

\section{Western blotting}

Proteins were resolved by polyacrylamide gel electrophoresis and transferred onto polyvinylidene difluoride (PVDF) membranes (Millipore, Germany). The membranes were blocked in Tris-buffered saline containing $0.2 \%$ Tween 20 and 5\% fat-free dry milk and incubated first with primary antibodies and then with horseradish peroxidase-conjugated secondary antibodies. Specific proteins were visualized with enhanced chemiluminescence detection reagent according to the manufacturer's instructions (Pierce Biotechnology, Waltham, MA, USA).

\section{MTS assay}

Cells grown in 96-well culture plates were treated with a gradient of concentrations of rapamycin or RAD001 for $72 \mathrm{~h}$. Cell growth inhibition was determined with the Cell Titer 96 Aqueous Non-Radioactive Cell Proliferation Assay (Promega, Fitchburg, WI, USA) according to the manufacturer's instructions.

\section{SRB assay}

Cells grown in 96-well culture plates were treated with a gradient of concentrations of rapamycin or RAD001 for $72 \mathrm{~h}$, fixed with $10 \%$ trichloroacetic acid and stained with $0.4 \%$ SRB dissolved in $1 \%$ acetic acid. The cells were then washed with $1 \%$ acetic acid to remove unbound dye. The protein-bound dye was extracted with $10 \mathrm{mmol} / \mathrm{L}$ Tris base to determine the optical density at a wavelength of $490 \mathrm{~nm}$. The percentage of cell proliferation as a function of drug concentration was plotted to determine the $\mathrm{IC}_{50}$, which represents the drug concentration needed for $50 \%$ inhibition of cell proliferation.

\section{Statistical analysis}

SPSS (Statistical Package for the Social Sciences) software version 17 (IBM Corporation, NY, USA) was used for statistical analysis of the experimental results. Most of our results were represented by at least three independent experiments and are presented as the mean \pm standard deviation (SD) of triplicate samples. Error bars represent standard deviations between experiments.

One-way analysis of variance and Student's $t$-test were used to determine statistical significance. $P<0.05$ was considered statistically significant.

\section{Results}

\section{LKB1 mutant NSCLC cells displayed higher mTOR activity}

According to previous studies, the cell lines H460 and A549 have a nonsense LKB1 mutation at codon 37, whereas H1792 and Calu-1 possess wild-type LKB1 ${ }^{[13]}$. LKB1 has been reported to negatively regulate $\mathrm{mTOR}$ activity; thus, we sought to compare the basal level of mTOR activity in various NSCLC cells with or without LKB1 inactivation. As shown in Figure 1, we found that LKB1 mutant cells (H460 and A549) displayed mTOR activity that was higher than in LKB1 wildtype cells (H1792 and Calu-1), as demonstrated by increased phosphorylation of S6K and 4EBP1, the two main substrates downstream of mTOR.

LKB1 inactivation does not increase the sensitivity to mTOR inhibitors

mTOR is a key regulator of cell growth; thus, we hypothesized
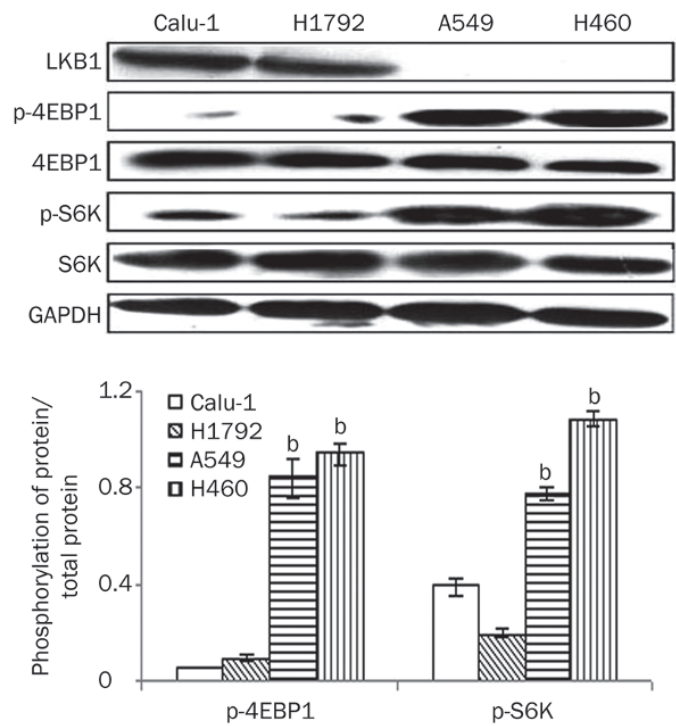

Figure 1. mTOR activity in various NSCLC cell lines. (A) p-S6K, S6K, p-4EBP1, and 4EBP1 were analyzed by Western blot. GAPDH served as a loading control. (B) Relative changes in p-S6K and p-4EBP1 protein expression in various groups. Statistical significance was evaluated relative to expression levels in LKB1 wild-type cells. ${ }^{\mathrm{b}} P<0.05$. 
that LKB1 mutant NSCLC cells with higher mTOR activity would be more sensitive to MTOR inhibitors than were LKB1 wild-type cells. To test this possibility, we evaluated the effect of mTOR inhibitors on the proliferation of these NSCLC cells. It was observed that treatment with $10 \mathrm{nmol} / \mathrm{L}$ rapamycin significantly decreased 4EBP1 phosphorylation in both LKB1 wild-type (H1792 and Calu-1) and LKB1 mutant cells (H460 and A549) (Figure 2A), confirming the inhibition of mTOR activity by rapamycin in NSCLC cells. We then treated these cells with a gradient of concentrations of rapamycin and measured cell proliferation by MTS assay. Unexpectedly, rapamycin had little effect on the growth of either H1299 (wild-type LKB1) or H460 (mutant LKB1) cells (Figure 2B). To validate our observation, we used the mTOR specific inhibitor RAD001. As shown in Figure 2C, treatment of A549 cells with a gradient of concentrations of RAD001 determined that RAD001 decreased 4EBP1 phosphorylation in a dose-dependent manner, indicating that it effectively inhibited mTOR activity. Next, we examined its effect on cell proliferation with MTS assay. Similarly to rapamycin, RAD001 did not obviously affect the proliferation of the various NSCLC cell lines (Figure 2D). Growth inhibition by RAD001, even at concentrations up to $1000 \mathrm{nmol} / \mathrm{L}$, was less than $50 \%$. Moreover, some LKB1 wild-type cells, such as Calu-1, were even more responsive to RAD001 than the LKB1 mutant cells, including H460. To confirm our results, we additionally measured cell proliferation by SRB assays, and similar results were achieved (Figure 2E). Thus, no association was found between sensitivity to mTOR inhibitors and LKB1 gene status.

To further verify our findings, we established the isogenic cell lines H1299-LKB1shRNA and H1299-PLKO.1 using a lentiviral short hairpin RNA against $\mathrm{LKB1}^{[12]}$. As shown in Figure $2 \mathrm{~F}$, LKB1 protein expression was significantly decreased in H1299-LKB1shRNA cells. We next examined the effect of rapamycin and RAD001 on the proliferation of H1299LKB1shRNA and H1299-PLKO.1 cells. As shown in Figure 2G and $2 \mathrm{H}$, stable knockdown of LKB1 did not significantly influence the growth inhibition induced by rapamycin or RAD001. Taken together, these data suggest that NSCLC cells are resistant to mTOR inhibitors and that LKB1 inactivation does not increase their sensitivity.

mTOR inhibition induces AKT activation in a PI3K-dependent manner

We next sought to examine the molecular mechanism underlying the resistance to mTOR inhibition in NSCLC cells with or without LKB1 inactivation. Because AKT signaling is known to be a major survival pathway, we examined the potential involvement of AKT activation in RAD001 treatment. NSCLC cells (H1792, Calu-1, A549, and H460) were treated with RAD001 (10 nmol/L) for $2 \mathrm{~h}$, and p-AKT was analyzed by Western blot. It was observed that RAD001 treatment led to a significant increase in $\mathrm{p}-\mathrm{AKT}$, suggesting that mTOR inhibition induced AKT activation (Figure 3A).

PI3K is the primary upstream regulator of AKT. To investigate whether PI3K is involved in RAD001-induced AKT activation, we treated H1792 and A549 cells with RAD001 (10 $\mathrm{nmol} / \mathrm{L}$ ) as a single agent or in combination with LY294002 (PI3K inhibitor, $10 \mu \mathrm{mol} / \mathrm{L}$ ) for $2 \mathrm{~h}$, and $\mathrm{p}$-AKT was analyzed by Western blot. We found that the combination of RAD001 with LY294002 attenuated the phosphorylation of AKT relative to the RAD001 alone treated group (Figure 3B). These data suggest that PI3K is involved in RAD001-induced AKT activation.

\section{Combination treatment with RAD001 and LY294002 enhances the antitumor effects.}

Because PI3K is required for RAD001-induced activation of AKT, we speculated that PI3K inhibition by LY294002 combined with RAD001 might increase growth inhibition. To investigate this possibility, H1792 and A549 cells were treated with RAD001 alone or in combination with LY294002 for $72 \mathrm{~h}$, and MTS assays were performed. As expected, the combination of RAD001 and LY294002 enhanced the antitumor effects (Figure 4A, 4B).

\section{Discussion}

A powerful approach to cancer therapy is to suppress oncogenic signaling using molecular-target inhibitors. RAD001 has been approved for the treatment of advanced renal carcinoma, pancreatic neuroendocrine cancers and certain cancers associated with hereditary tuberous sclerosis ${ }^{[14]}$. However, clinical trials indicate that RAD001 has only moderate antitumor efficacy ${ }^{[15,16]}$, and there remains an urgent need to identify predictive markers for selecting patients who will significantly benefit from treatment with rapalogs. Several studies have revealed that the PI3K/AKT/PTEN status might not be predictive of the rapalog response ${ }^{[17]}$. Shackelford found that rapamycin monotherapy decreased polyp burden and size in $\mathrm{LKB}^{+/-}$mice with polyposis ${ }^{[18]}$. In addition, endometrial cancers with LKB1 inactivation are highly responsive to mTOR inhibitors ${ }^{[11]}$, which led us to speculate that LKB1 may be a predictor of response to rapalogs. However, in the present study, we found that NSCLC cells are insensitive to rapamycin or RAD001 by both MTS and SRB assays, and the cellular response to $\mathrm{mTOR}$ inhibitors has no association with LKB1 gene status. Thus, LKB1 is not an appropriate predictor in NSCLC cell lines. As the signaling network of mTOR is quite complex, investigating additional biomarkers will be necessary in the future.

Previous studies have shown that rapamycin leads to a marked increase in AKT activity in MCF-7 (breast cancer cell line), MDA-MB-468 (breast cancer cell line) and DU-145 (prostate cancer cell line) cells ${ }^{[19,20]}$. Accordingly, we also found that mTOR inhibitors activate a negative feedback pathway, leading to activation of AKT, which might attenuate its anticancer effects. In addition, our data shows that the combined treatment of RAD001 with LY294002 decreased p-AKT, suggesting the involvement of PI3K in AKT activation induced by mTOR inhibition. This finding is in good agreement with a study showing that the inhibition of p70/S6K induced by rapamycin results in increased IGF-IR/IRS-1/PI3K signaling to phosphor- 
A
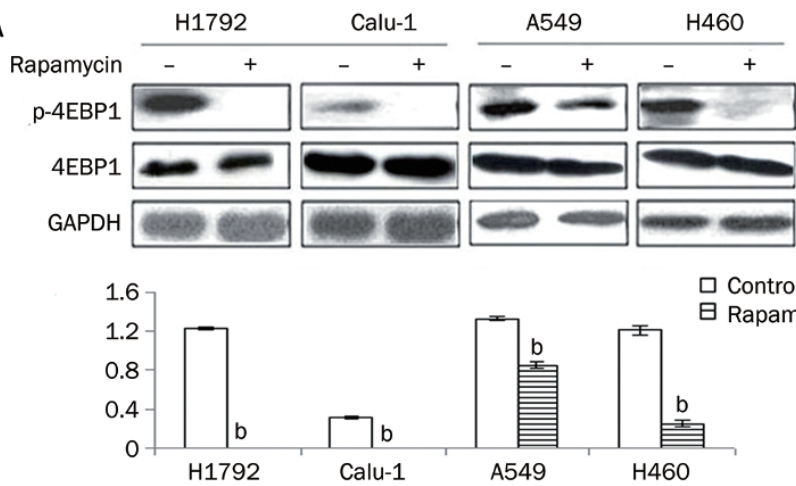

C
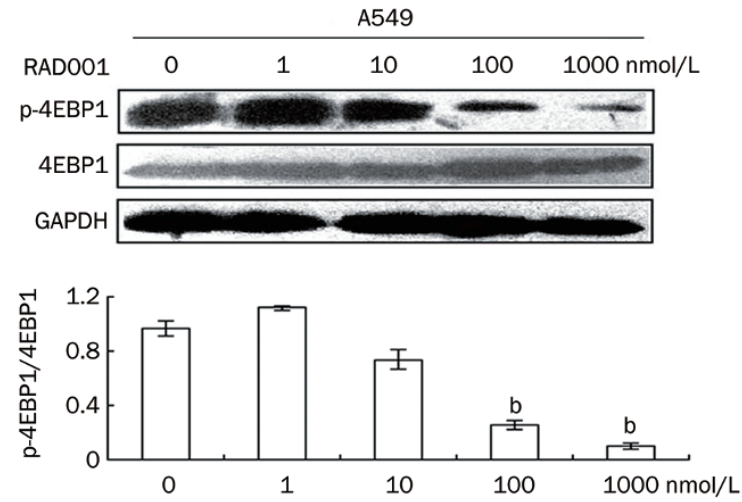

E

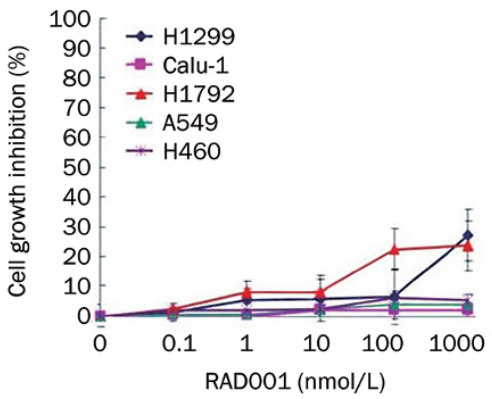

G

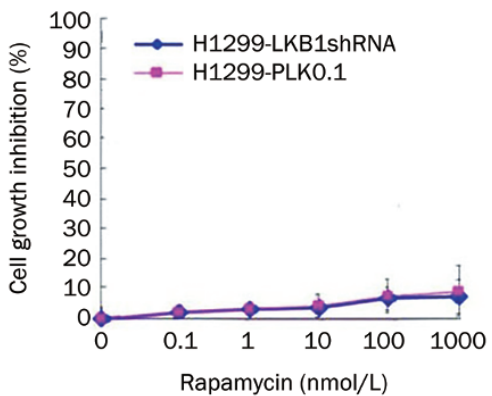

B

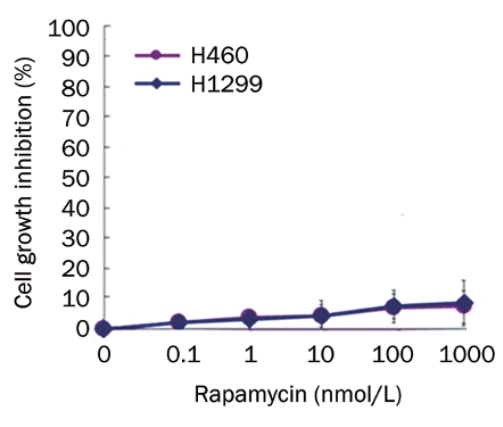

D

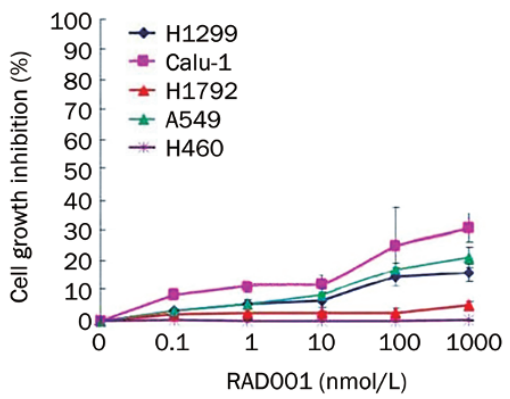

F

H1299-PLK0.1 H1299-LKB1shRNA

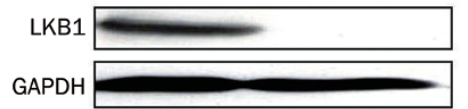

$\mathrm{H}$

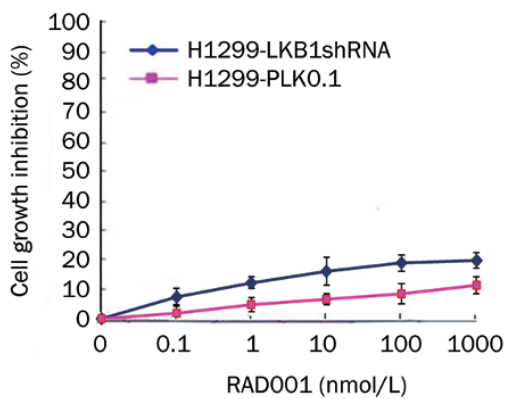

Figure 2. LKB1 inactivation does not increase the sensitivity to mTOR inhibitors. (A) H1792, Calu-1, A549, and H460 cells were treated with 10 nmol/L rapamycin for $2 \mathrm{~h}$. Cell extracts were subjected to immunoblotting analysis for p-4EBP1, 4EBP1, and GAPDH. Statistical significance was evaluated by comparison with the control group using Student's $t$-test. ${ }^{b} P<0.05$. (B) $\mathrm{H} 1299$ and $\mathrm{H} 460$ were seeded on 96 -well plates and exposed to rapamycin at different concentrations. The cells were subjected to MTS assays after $72 \mathrm{~h}$. (C) A549 cells were exposed to RAD001 (0, 1, 10, 100, and 1000 $\mathrm{nmol} / \mathrm{L}$ ) for $2 \mathrm{~h}$. p-4EBP1, 4EBP1, and GAPDH were tested by immunoblotting analysis. Statistical significance was evaluated using one-way analysis of variance. ${ }^{b} \mathrm{P}<0.05$. (D) H1299, H1792, Calu-1, H460, and A549 cells were treated with RAD001 (0, 0.1, 1, 10, 100, and 1000 nmol/L) and were subjected to an MTS assay after $72 \mathrm{~h}$. (E) RAD001 was treated in the same cell line with concentrations of 0, 0.1, 1, 10, 100, and 1000 nmol/L and were then subjected to SRB assays after 72 h. (F) Anti-LKB1 antibody was used to detect LKB1 using GAPDH as the loading control. (G) H1299LKB1shRNA and H1299-PLKO.1 were treated with a gradient of concentrations of rapamycin $(0,0.1,1,10,100$, and $1000 \mathrm{nmol} / \mathrm{L})$ for $72 \mathrm{~h}$, and MTS assays were performed to measure cell proliferation. (H) H1299-LKB1shRNA and H1299-PLKO.1 were treated with a gradient of concentrations of $\operatorname{RAD001}(0,0.1,1,10,100$, and $1000 \mathrm{nmol} / \mathrm{L})$ for $72 \mathrm{~h}$, and MTS assays were performed to measure cell proliferation. 
A
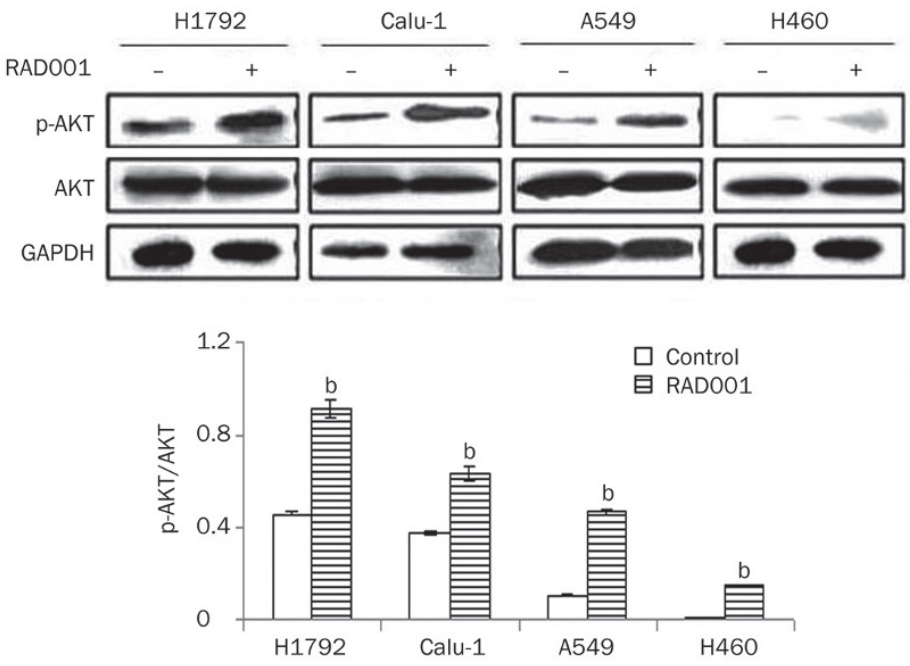

B
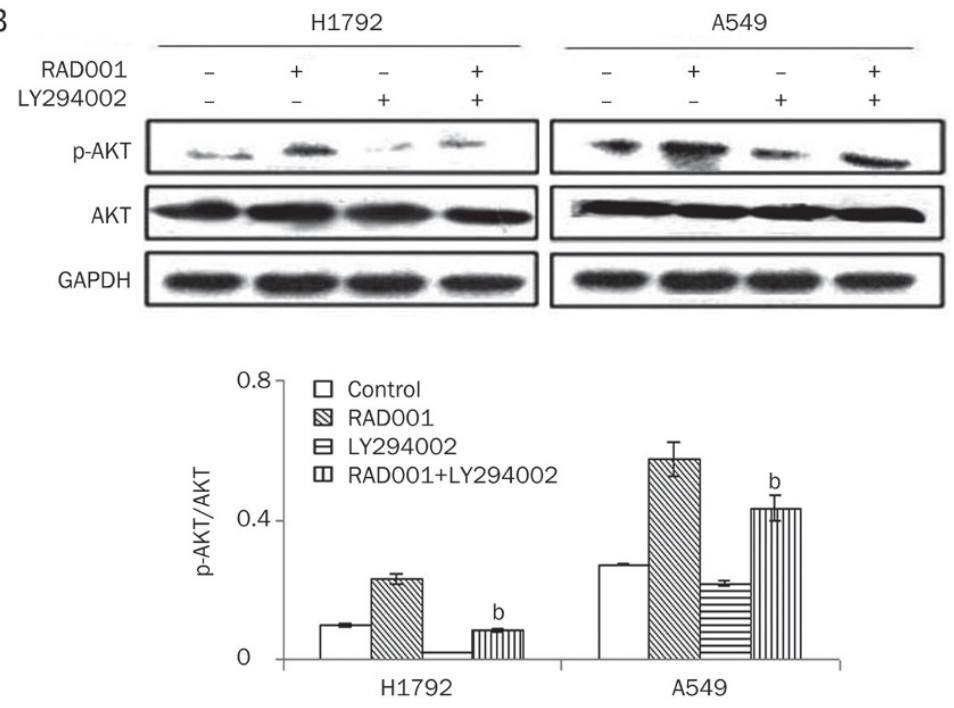

Figure 3. Effect of RAD001 on AKT phosphorylation. (A) H1792, Calu-1, A549, and H460 cells were treated with $10 \mathrm{nmol} / \mathrm{L}$ RAD001 for $2 \mathrm{~h}$. p-AKT and AKT were analyzed by Western blot using GAPDH as a loading control. Relative changes in p-AKT protein expression were tested in various groups. Statistical significance was evaluated by comparison with the control group using Student's $t$ test. ${ }^{b} P<0.05$ vs control. (B) H1792 and A549 cells were treated with the control, RAD001 (10 nmol/L) alone, LY294002 (10 $\mu \mathrm{mol} / \mathrm{L})$ alone and LY294002 (10 $\mu \mathrm{mol} / \mathrm{L})$ in combination with RAD001 (10 nmol/L) for $2 \mathrm{~h}$. p-AKT and AKT were analyzed by Western blot. GAPDH was served as the loading control. Statistical significance was evaluated using one-way analysis of variance. ${ }^{\mathrm{b}} \mathrm{P}<0.05$ vs RAD001 alone.

ylate $\mathrm{AKT}^{[19]}$. Thus, mTOR/PI3K dual inhibitors may synergistically inhibit tumorigenesis, as evidenced in our experiment. However, the precise mechanism by which an mTOR inhibitor induces AKT activation remains to be determined. It has been reported that rapamycin increases the expression of receptor tyrosine kinases, such as EGFR, HER2, and HER3, which might lead to feedback activation of $\mathrm{AKT}^{[21,22]}$. Future studies are needed to clarify these questions.

In conclusion, our study demonstrates that LKB1 inactivation does not affect the cellular response to mTOR inhibitors in non-small cell lung cancer cells. In addition, we found that mTOR inhibitors induced negative feedback activation of AKT, which might contribute to the observed resistance.

\section{Acknowledgements}

This study was supported by grants from the National Natural Science Foundation of China (81071915 and 31301160) and the Tianjin Health Bureau (2011KZ107).

\section{Author contribution}

Dr Dian-sheng ZHONG initiated the project; Ping XIAO, Lin-lin SUN, and Jing WANG designed and performed the research; Rui-li HAN, Qing MA analyzed the data; Ping XIAO, Lin-lin SUN, and Dian-sheng ZHONG wrote the manuscript.

\section{References}

1 Siegel R, Naishadham D, Jemal A. Cancer statistics, 2012. CA 
A

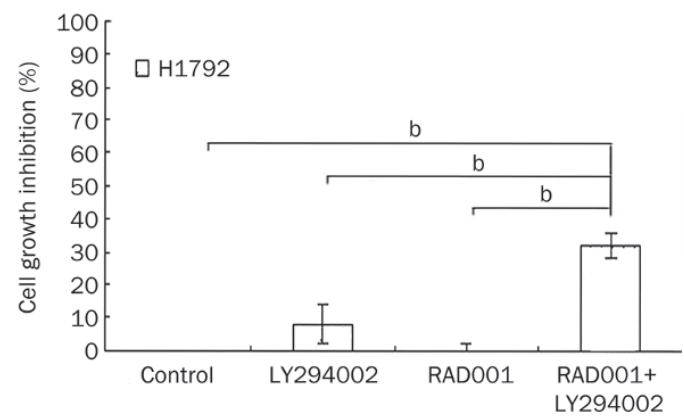

B

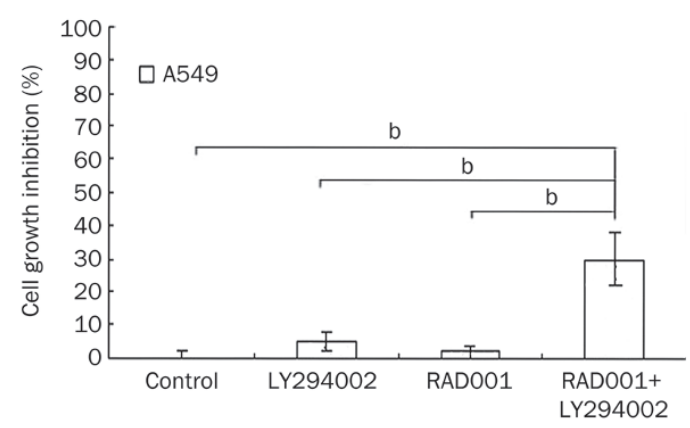

Figure 4. Combined PI3K and mTOR inhibitors synergistically inhibited cell growth. Cell growth inhibition was tested by treatment with LY294002 $(10 \mu \mathrm{mol} / \mathrm{L})$ or RAD001 (10 $\mathrm{nmol} / \mathrm{L})$ as single agents or with combined LY294002 (10 $\mathrm{mmol} / \mathrm{L})$ and RAD001 $(10 \mathrm{nmol} / \mathrm{L})$ for $72 \mathrm{~h}$. Statistical significance was evaluated using one-way analysis of variance. ${ }^{\mathrm{b}} P<0.05$.

Cancer J Clin 2012; 62: 10-29.

2 Vaahtomeri K, Mäkelä TP. Molecular mechanisms of tumor suppression by LKB1. FEBS Lett 2011; 585: 944-51.

3 Shah U, Sharpless NE, Hayes DN. LKB1 and lung cancer: more than the usual suspects. Cancer Res 2008; 6810: 3562-5.

4 Herrmann JL, Byekova Y, Elmets CA, Athar M. Liver Kinase B1 (LKB1) in the pathogenesis of epithelial cancers. Cancer Lett 2011; 306: 1-9.

5 Inoki K, Zhu T, Guan KL. TSC2 mediates cellular energy response to control cell growth and survival. Cell 2003; 115: 577-90.

6 Holz MK, Ballif BA, Gygi SP, Blenis J. mTOR and S6K1 mediate assembly of the translation preinitiation complex through dynamic protein interchange and ordered phosphorylation events. Cell 2005; 123: 569-80.

7 Rizzieri DA, Feldman E, DiPersio JF, Gabrail N, Stock W, Strair R, et al. A phase 2 clinical trial of deforolimus (AP23573, MK-8669), a novel mammalian target of rapamycin inhibitor, in patients with relapsed or refractory hematologic malignancies. Clin Cancer Res 2008; 14: 2756-62.

8 Rini BI. Temsirolimus, an inhibitor of mammalian target of rapamycin. Clin Cancer Res 2008; 14: 1286-90.

9 Wolpin BM, Hezel AF, Abrams T, Blaszkowsky LS, Meyerhardt JA, Enzinger PC, et al. Oral mTOR inhibitor everolimus in patients with gemcitabine-refractory metastatic pancreatic cancer. J Clin Oncol 2009; 27: 193-8.

10 Xu G, Zhang W, Bertram P, Zheng XF, McLeod H. Pharmacogenomic profiling of the PI3K/PTEN-AKT-mTOR pathway in common human tumors. Int J Oncol 2004; 24: 893-900.

11 Contreras CM, Akbay EA, Gallardo TD, Haynie JM, Sharma S, Tagao O, et al. Lkb1 inactivation is sufficient to drive endometrial cancers that are aggressive yet highly responsive to mTOR inhibitor monotherapy. Dis Model Mech 2010; 3: 181-93.

12 Sun L, Zhong D, Wu S, Bai H, Chen Z. Establishment and gene expression profiling of LKB1 stable knockdown lung cancer cell line. Chin Med J(Engl) 2011; 124: 2028-32.

13 Zhong D, Guo L, de Aguirre I, Liu X, Lamb N, Sun SY, et al. LKB1 mutation in large cell carcinoma of the lung. Lung Cancer 2006; 53: 285-94.

14 Nyfeler B, Chen Y, Li X, Pinzon-Ortiz M, Wang Z, Reddy A, et al. RAD001 enhances the potency of BEZ235 to inhibit mTOR signaling and tumor growth. PLoS One 2012; 7: e48548.

15 Zhu AX, Abrams TA, Miksad R, Blaszkowsky LS, Meyerhardt JA, Zheng $\mathrm{H}$, et al. Phase $1 / 2$ study of everolimus in advanced hepatocellular carcinoma. Cancer 2011; 117: 5094-102.

16 Rizell M, Andersson M, Cahlin C, Hafstrom L, Olausson M, Lindner P. Effects of the mTOR inhibitor sirolimus in patients with hepatocellular and cholangiocellular cancer. Int Clin Oncol 2008; 13: 66-70.

17 Meric-Bernstam F, Gonzalez-Angulo AM. Targeting the mTOR signaling network for cancer therapy. J Clin Oncol 2009; 27: 2278-87.

18 Shackelford DB, Shaw RJ. The LKB1-AMPK pathway: metabolism and growth control in tumour suppression. Nat Rev Cancer 2009; 9: 563-75.

19 O'Reilly KE, Rojo F, She QB, Solit D, Mills GB, Smith D, et al. mTOR inhibition induces upstream receptor tyrosine kinase signaling and activates Akt. Cancer Res 2006; 66: 1500-8.

20 Sun SY, Rosenberg LM, Wang X, Zhou Z, Yue P, Fu H, et al. Activation of Akt and elF4E survival pathways by rapamycin-mediated mammalian target of rapamycin inhibition. Cancer Res 2005; 65: 7052-8.

21 Chandarlapaty S, Sawai A, Scaltriti M, Rodrik-Outmezquine V, GrbovicHuezo O, Serra V, et al. AKT inhibition relieves feedback suppression of receptor tyrosine kinase expression and activity. Cancer Cell 2011; 19: $58-71$

22 Rodrik-Outmezguine VS, Chandarlapaty S, Pagano NC, Poulikakos $\mathrm{PI}$, Scaltriti M, Moskatel E, et al. mTOR kinase inhibition causes feedback-dependent biphasic regulation of AKT signaling. Cancer Discov 2011; 1: 248-59. 